\title{
Heated vegetable oils and cardiovascular disease risk factors
}

\begin{abstract}
Cardiovascular disease (CVD) is one of the leading major causes of morbidity and mortality worldwide. It may result from the interactions between multiple genetic and environmental factors including sedentary lifestyle and dietary habits. The quality of dietary oils and fats has been widely recognised to be inextricably linked to the pathogenesis of CVD. Vegetable oil is one of the essential dietary components in daily food consumption. However, the benefits of vegetable oil can be deteriorated by repeated heating that leads to lipid oxidation. The practice of using repeatedly heated cooking oil is not uncommon as it will reduce the cost of food preparation. Thermal oxidation yields new functional groups which may be potentially hazardous to cardiovascular health. Prolonged consumption of the repeatedly heated oil has been shown to increase blood pressure and total cholesterol, cause vascular inflammation as well as vascular changes which predispose to atherosclerosis. The harmful effect of heated oils is attributed to products generated from lipid oxidation during heating process. In view of the potential hazard of oxidation products, therefore this review article will provide an insight and awareness to the general public on the consumption of repeatedly heated oils which is detrimental to health.
\end{abstract}

Keyword: Plant oils; Heating; Cardiovascular diseases; Aetiology 